\title{
Dermatomiositis y cáncer colorrectal: reporte de caso y revisión de la literatura
}

\section{Dermatomyositis and colorectal cancer: Case report and literature review}

Mario Andrés Jaramillo-Santos, ${ }^{1}$ (D) Andrés Sánchez-Gil,, ${ }^{2}$ (D) Andrés Valencia-Uribe, ${ }^{3 *}$ (D) Lázaro Arango-Molano. ${ }^{4}$ (1)

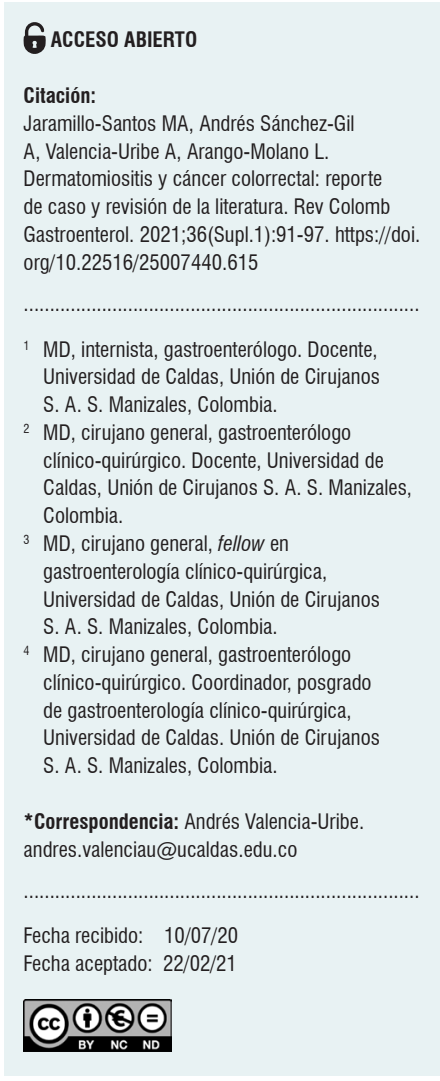

\section{Resumen}

Los pacientes con dermatomiositis presentan un mayor riesgo de asociación con patología maligna subyacente, sin conocerse una causa precisa de esta relación. Son múltiples las posibles localizaciones anatómicas, entre las que se incluyen los ovarios, mamas, zona gástrica, zona colorrectal, sangre, pulmones y próstata.

Se presenta el caso de una mujer de 58 años que durante el estudio de pérdida anormal de peso manifestó debilidad muscular grave y alteraciones dermatológicas asociadas con el hallazgo de adenocarcinoma del colon transverso.

\section{Palabras clave}

Dermatomiositis, malignidad, cáncer colorrectal, síndrome paraneoplásico.

\section{Abstract}

Patients with dermatomyositis are more likely to have an underlying malignancy, although the exact cause of this association is unknown. There are multiple possible anatomical sites, including ovaries, breasts, stomach, colorectum, blood, lungs, and prostate.

We present the case of a 58-year-old woman who during abnormal weight loss study showed severe muscle weakness and skin alterations, associated with finding of adenocarcinoma of the transverse colon.

\section{Keywords}

Dermatomyositis: Malignancy; Colorectal cancer; Paraneoplastic syndrome.

\section{INTRODUCCIÓN}

La dermatomiositis es una enfermedad del colágeno ampliamente estudiada, caracterizada por debilidad mus- cular y asociada con lesiones cutáneas. Existe una mayor frecuencia de malignidad en los pacientes con dermatomiositis que en la población general. Es frecuente que la dermatomiositis sea de causa paraneoplásica y las altas 
tasas de detección de cáncer tras el diagnóstico de dermatomiositis hacen necesaria la investigación con respecto a la búsqueda del mismo, teniendo en cuenta el grupo etario y la clínica de cada individuo (1-3). Adicionalmente, se ha descrito como una manifestación paraneoplásica de patología maligna gastrointestinal.

Los cánceres más frecuentemente asociados con dermatomiositis son el ovárico, pulmonar, de mama, gástrico, colorrectal, pancreático y el linfoma no hodgkiniano (3-6).

\section{CASO CLÍNICO}

Se trata de una mujer de 58 años de edad quien consultó por un cuadro de pérdida anormal de peso de $6 \mathrm{~kg}$ en el último semestre, asociado con hematoquecia escasa y deposiciones diarreicas ocasionales, sin antecedentes patológicos, con antecedente familiar de cáncer de colon (hermana durante la quinta década de la vida). Se realizó una colonoscopia total que demostró tres pólipos: uno en el colon ascendente de $7 \mathrm{~mm}$ (biopsia: adenoma velloso con displasia de bajo grado) (Figura 1), un pólipo sésil de $50 \mathrm{~mm}$ en ángulo hepático (biopsia: adenoma velloso con displasia de bajo grado) (Figura 2) y un pólipo de $30 \mathrm{~mm}$ en el recto superior (biopsia: adenoma con displasia de bajo grado) (Figura 3); el hemograma resultó normal en las 3 líneas celulares. Se llevó a polipectomía endoscópica de pólipo rectal tipo piece meal con asa caliente, cuyo estudio histopatológico reportó adenoma tubulovelloso con displasia de bajo grado.

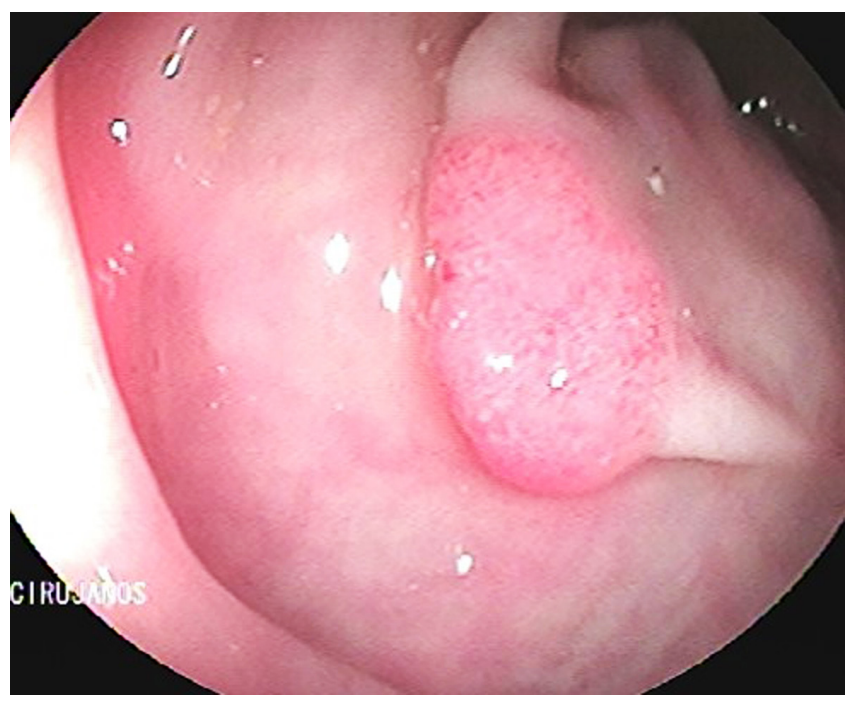

Figura 1. Pólipo sésil del colon ascendente de $7 \mathrm{~mm}$.

Un mes después, por el pólipo descrito en el ángulo hepático sin posibilidad de resección endoscópica, se realizó una hemicolectomía derecha e ileotransversosto- mía, con un reporte histopatológico de 2 adenomas tubulares con displasia de alto grado focal sin compromiso pedicular, bordes de resección libres de lesión y sin compromiso ganglionar. 2 meses después, en la colonoscopia total de seguimiento se encontró, además de los cambios posquirúrgicos, un pólipo sésil de $40 \times 20 \mathrm{~mm}$ en el colon transverso inmediatamente distal a la anastomosis (biopsia: adenoma velloso con displasia de bajo grado) (Figura 4); debido a este hallazgo, se llevó a una nueva cirugía con ampliación de la colectomía, omentectomía y nueva anastomosis ileocolónica con un reporte histopatológico de adenoma tubulovelloso con displasia de alto grado y adenocarcinoma in situ inmediatamente distal a la anastomosis previa, sin signos de infiltración, bordes de resección libres, sin compromiso ganglionar ni del omento mayor. 6 meses después se realizó una nueva ileocolonoscopia con un único hallazgo de pólipo de $4 \mathrm{~mm}$ en el recto superior resecado con asa fría (patología: adenoma tubulovelloso con displasia de bajo grado) (Figura 5).

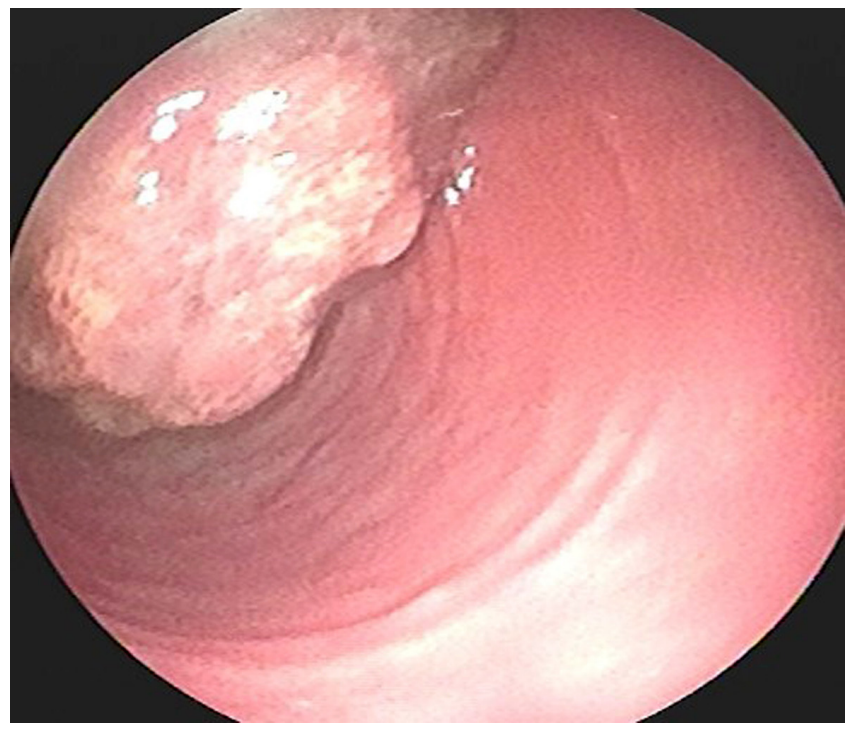

Figura 2. Pólipo sésil de 50 mm en ángulo hepático del colon.

4 meses antes de la intervención quirúrgica inicial, la paciente presentó máculas cutáneas eritematosas en la cara anterior del tórax, que se hicieron más intensas, de mayor tamaño y se extendieron a la región dorsolumbar, miembros superiores y cara (Figuras 6-11). 2 meses después desarrolló debilidad muscular generalizada con caídas desde la bipedestación e incapacidad para reincorporarse, asociada con edema y dolor distal en extremidades superiores e inferiores. Se evaluaron niveles séricos de creatinacinasa (CK) total iniciales de $2028 \mu \mathrm{mol} / \mathrm{L}$ y luego de la resección quirúrgica ampliada se evidenció un descenso progresivo a 1800 y $1028 \mu \mathrm{mol} / \mathrm{L}$, con mejoría en los síntomas. Las biopsias de piel de la región pectoral derecha y 


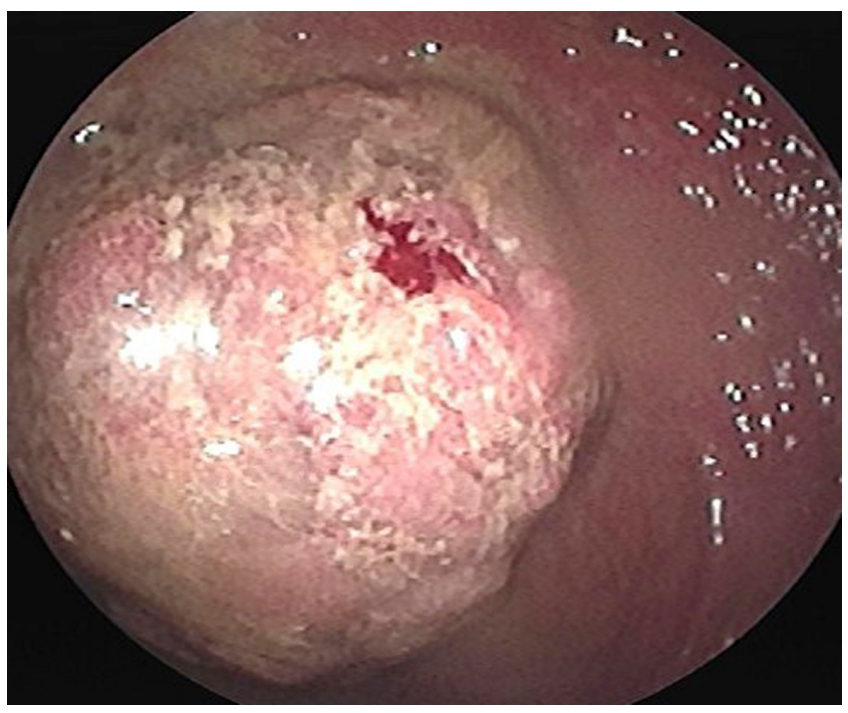

Figura 3. Pólipo de $30 \mathrm{~mm}$ en recto superior.

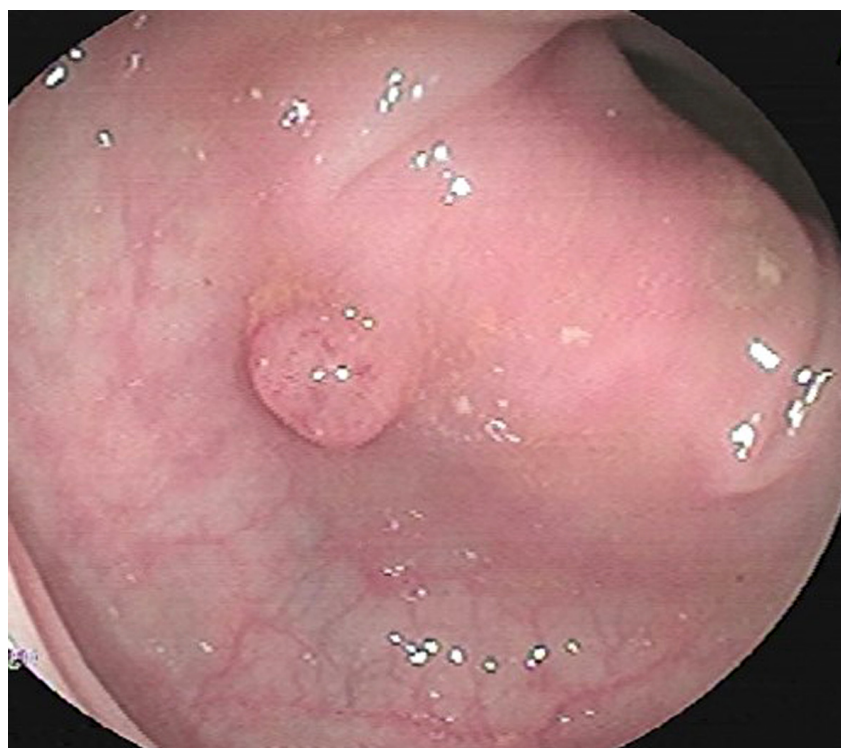

Figura 5. Pólipo de $4 \mathrm{~mm}$ en recto superior.

el brazo derecho reportaron dermatitis crónica de interfase con cambios vacuolares compatibles con dermatomiositis. Con estos hallazgos y comportamientos clínicos, de laboratorio e histopatológicos se concluyó que la paciente cursaba con dermatomiositis como manifestación paraneoplásica del cáncer de colon. Los marcadores tumorales (Ca 19,9 y ACE séricos) fueron normales. La tomografía axial computarizada (TAC) de tórax y abdominopélvica contrastada resultaron sin signos sugestivos de secundarismo.

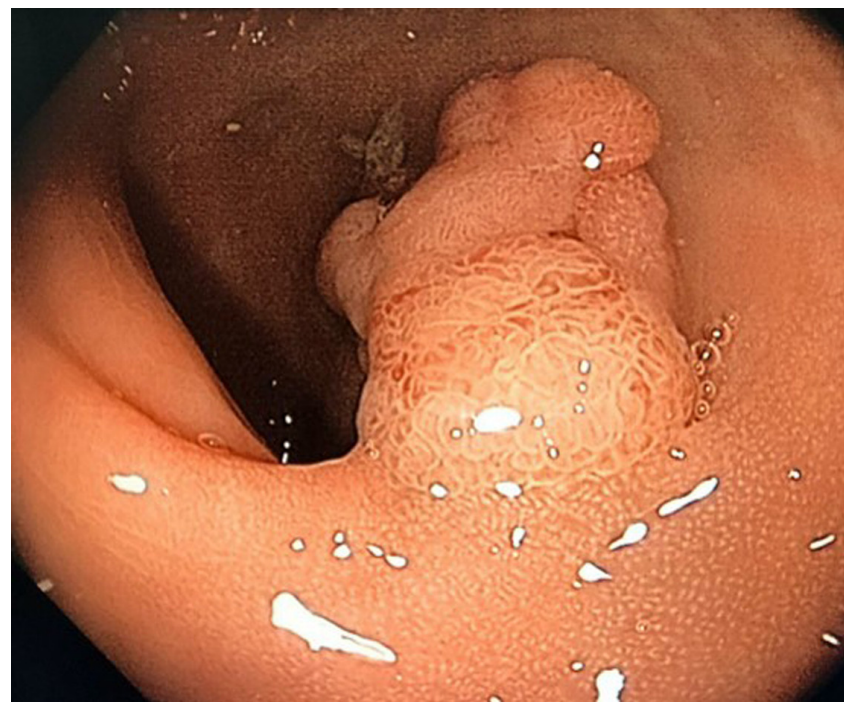

Figura 4. Pólipo sésil de 40 × 20 mm en colon transverso.

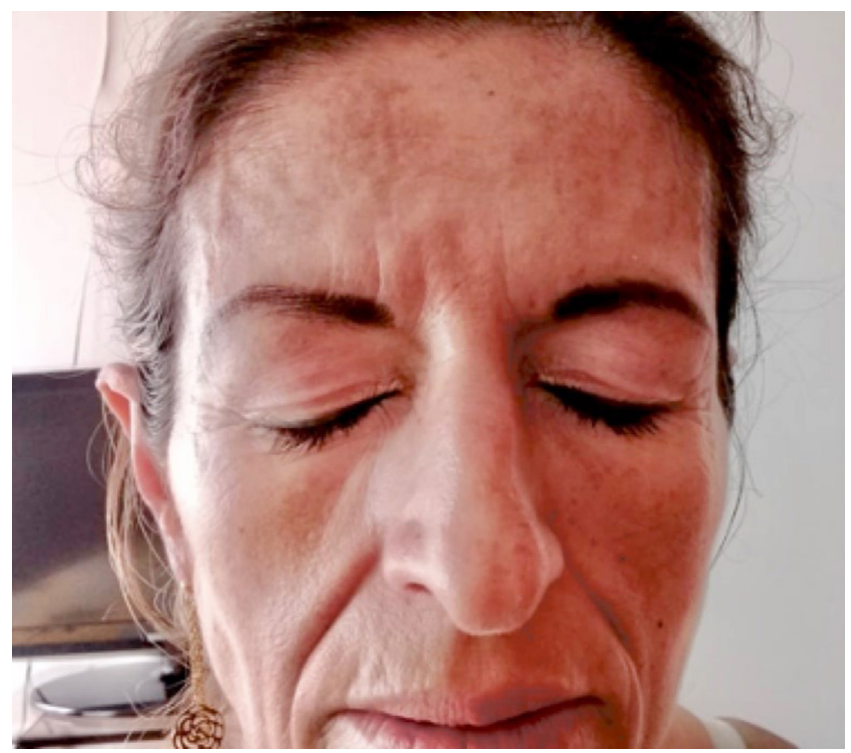

Figura 6. Eritema bipalpebral y facial a nivel frontal, interciliar y en las mejillas.

Actualmente, la paciente está en manejo por reumatología y dermatología, con esteroides sistémicos con resolución de los síntomas musculares y mejoría significativa de las lesiones dermatológicas (Figura 12).

\section{DISCUSIÓN}

Las dermatomiositis pertenecen a un grupo heterogéneo de enfermedades del tejido conectivo llamado miopatías 


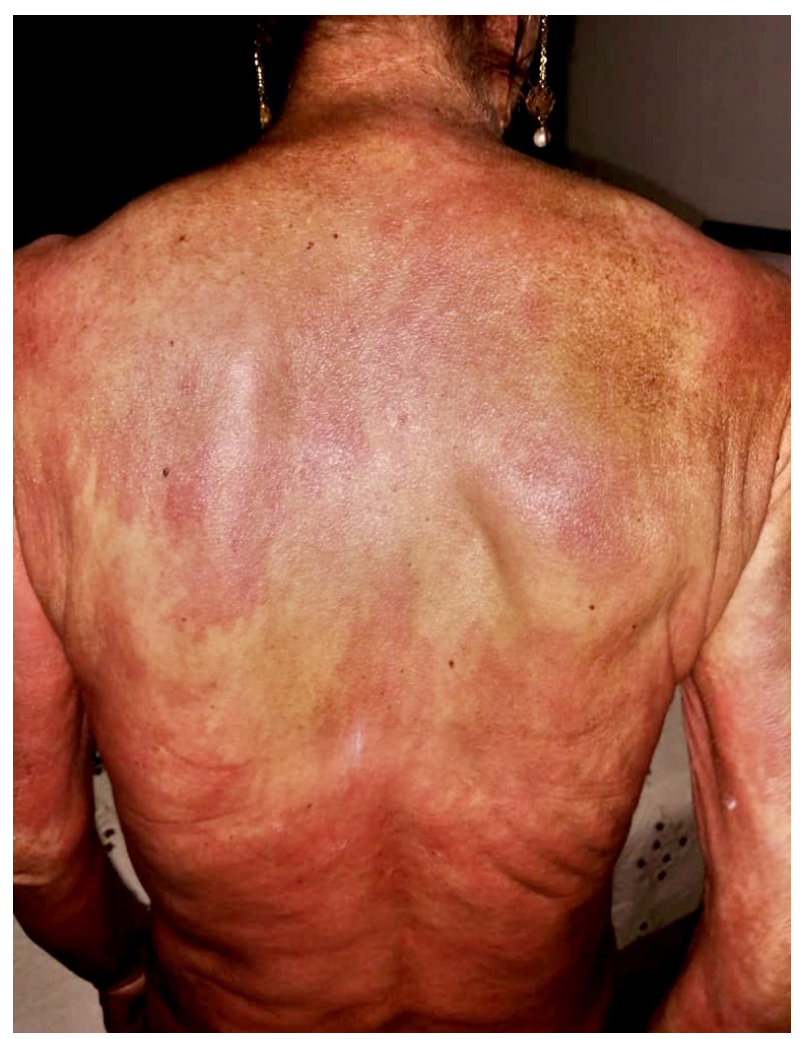

Figura 7. Placas eritematosas en la región cervical posterior, dorsal y en los hombros, que configura el signo del chal o eritema en capelina.

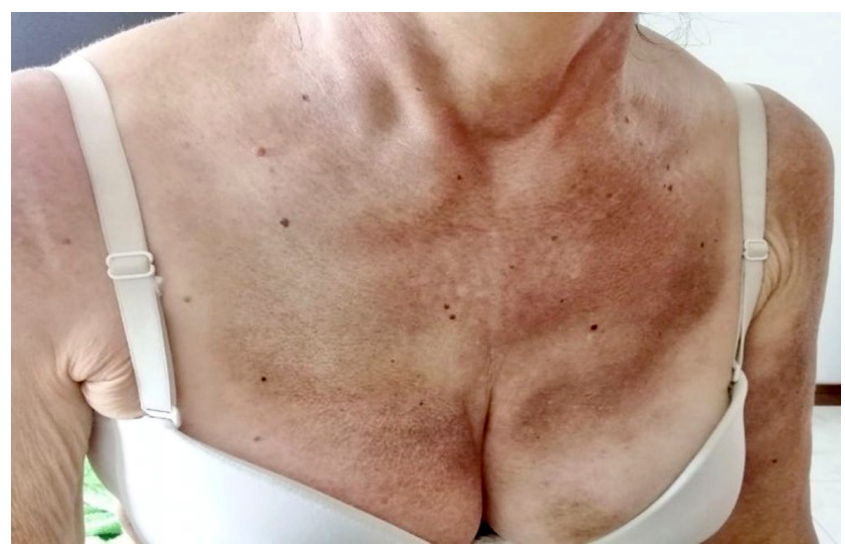

Figura 9. Placas eritematovioláceas en la región anterior del cuello con extensión al tórax anterior.

inflamatorias idiopáticas (7). Esta entidad se presenta clínicamente con debilidad e inflamación musculoesquelética proximal, y manifestaciones dermatológicas. Su incidencia en Estados Unidos es de 5 a 10 por 1 millón de habitantes $y$ tiene predilección por el sexo femenino en relación 3:1. El

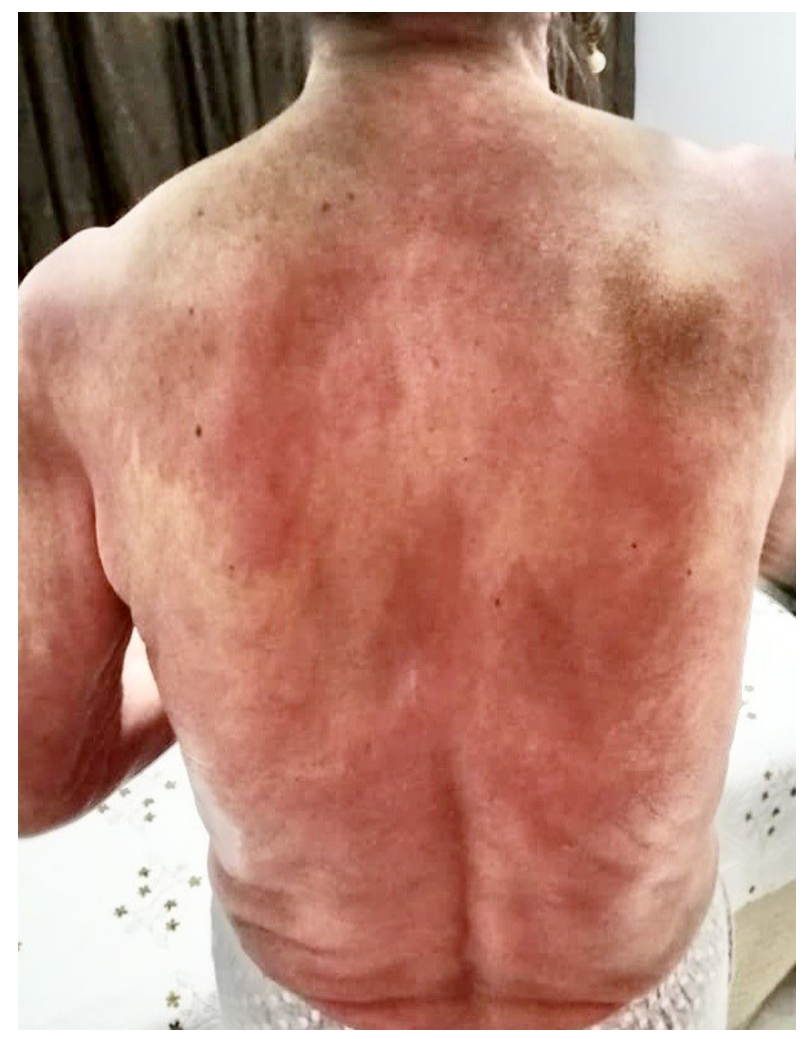

Figura 8. Placas eritematosas que se extienden hasta la región lumbar.

pico de incidencia se da entre los 40 y 60 años de edad (8). La asociación de dermatomiositis y malignidad fue establecida por primera vez en 1916 en un paciente con cáncer gástrico. Desde entonces, múltiples series de casos retrospectivas han encontrado la asociación entre malignidad y dermatomiositis en aproximadamente el $24 \%$ de los casos (9).

La etiología de la dermatomiositis asociada con cáncer permanece desconocida, aunque se han propuesto varias hipótesis como la liberación de componentes bioactivos que generan reacciones inmunitarias en las fibras musculares y la piel (10). Se ha encontrado que las fibras musculares afectadas por patología autoinmunitaria sobreexpresan autoantígenos específicos y que esta sobreexpresión es exhibida por células tumorales y mioblastos en regeneración, lo que indica que existe similitud entre los antígenos expresados por ambos tipos de células (11). Se plantea que hay una respuesta inmunitaria cruzada en contra de las células musculares en regeneración, lo que lleva a desarrollar la enfermedad $(12,13)$.

Los criterios diagnósticos de la dermatomiositis fueron establecidos en 1975 por Boham y Peter (14) del siguiente modo: debilidad muscular, exámenes de laboratorio con elevación del valor serológico de las enzimas musculares, 


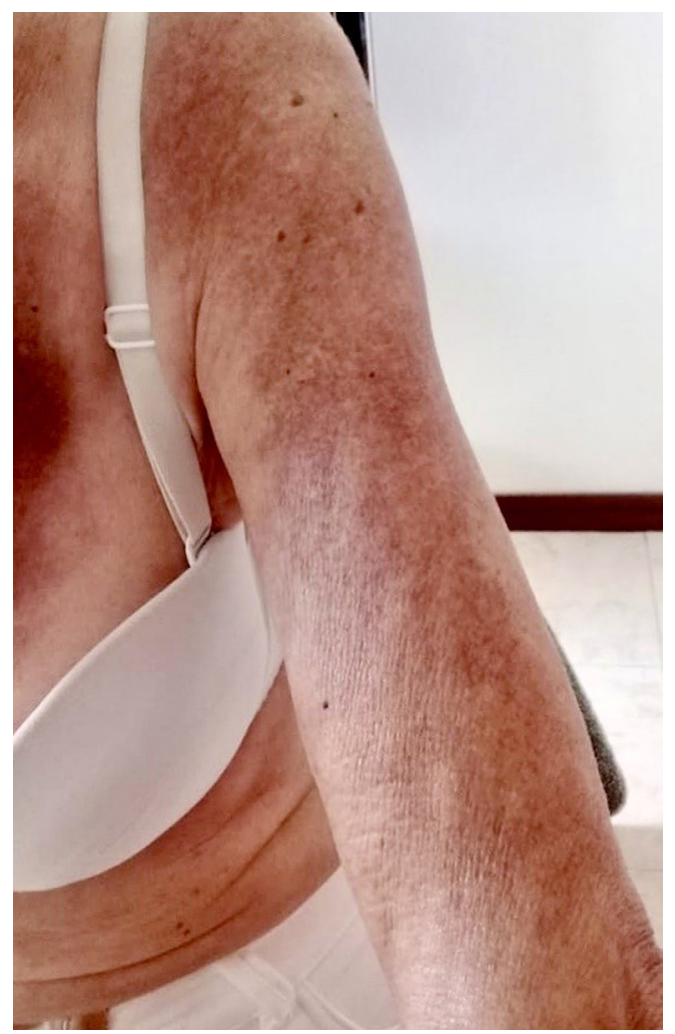

Figura 10. Placas eritematovioláceas en la cara anterior del hombro y brazos.

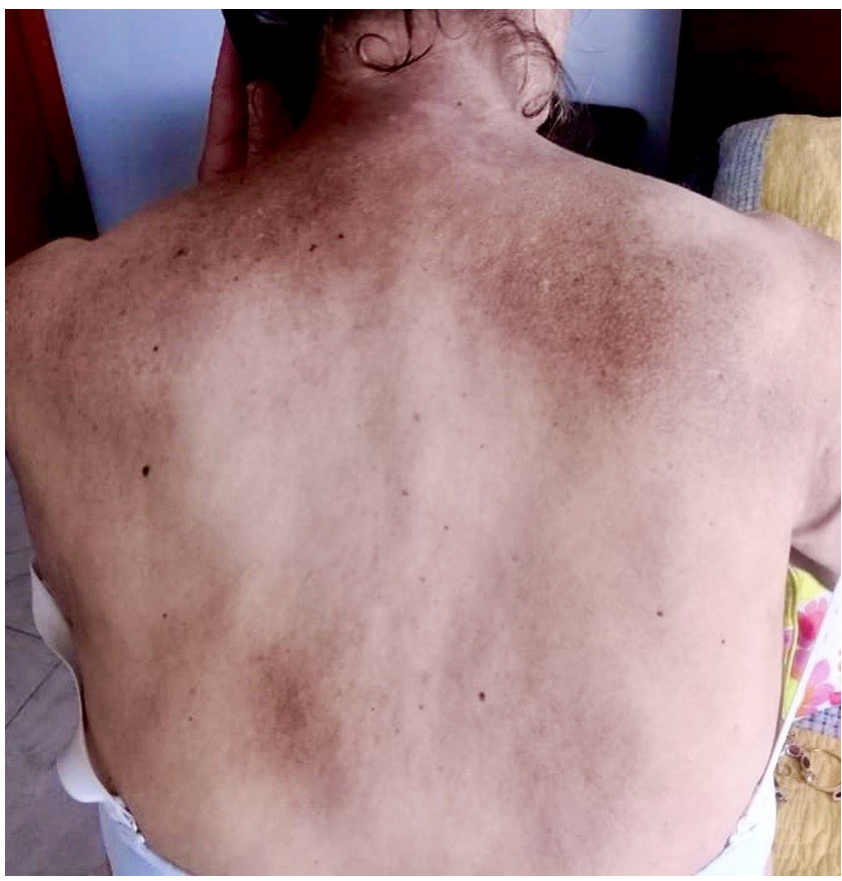

Figura 12. Mejoría de las lesiones cervicales, dorsales y lumbares.

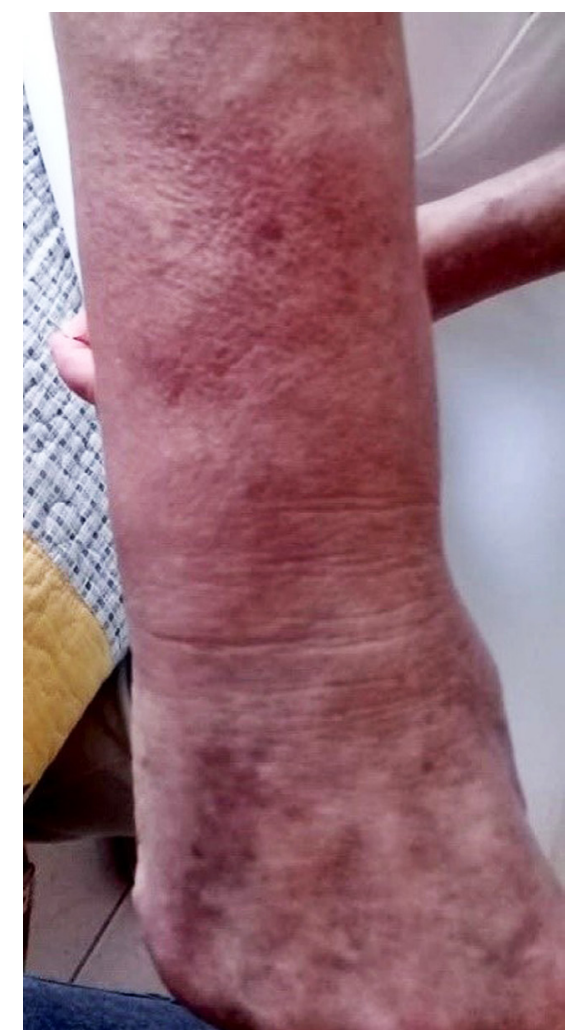

Figura 11. Compromiso distal de los miembros superiores dado por placas eritematovioláceas que comprometen el dorso de las manos.

hallazgos anormales en la electromiografía que indican miopatía, estudio patológico de biopsia muscular o de la piel compatible, y manifestaciones cutáneas. El diagnóstico de dermatomiositis se establece con la presencia de lesiones cutáneas características asociada con 3 o más de los demás criterios. Cuando no hay debilidad muscular, el diagnóstico se denomina dermatomiositis amiopática (15), que hace parte del mismo espectro de síndrome paraneoplásico $(16,17)$.

Leatham y colaboradores (18) en un análisis retrospectivo de pacientes con dermatomiositis en Estados Unidos (400 casos), encontraron malignidad confirmada en el $15,8 \%$ delos casos, delos siguientes tipos: de mama $(24,5 \%)$, hematológica $(17 \%)$, colorrectal $(9,4 \%)$ y de próstata $(9,4 \%)$. Hill y colaboradores (4) encontraron una asociación de cáncer con dermatomiositis en orden decreciente de frecuencia: ovario, pulmón, estómago, colorrectal, páncreas y linfoma no hodgkiniano. Sigurgeirsson y colaboradores (19) reportaron los cánceres colorrectal y pulmonar como los más frecuentes en una serie de 750 pacientes con dermatomiositis y polimiositis. Hatada y colaboradores (20) en Japón encontraron que la malignidad más frecuente en 
dermatomiositis es gástrica $(25,4 \%)$. En todas las series, los cánceres del tracto digestivo hacen parte de las malignidades más frecuentes en pacientes con dermatomiositis. Varios estudios han reportado un riesgo incrementado de desarrollar cáncer colorrectal en personas con dermatomiositis $(4,19,21-23)$.

La literatura es ambigua en cuanto a la presencia de predictores clínicos o paraclínicos de malignidad en pacientes con dermatomiositis. Se ha propuesto la presencia de neumonitis intersticial, niveles séricos elevados de CK, necrosis cutánea (úlceras), poiquilodermia, velocidad de sedimentación globular (VSG) $>40 \mathrm{~mm} / \mathrm{h}$, proteína C-reactiva (PCR) elevada, hipoalbuminemia y edad $>50$ años (17, 24-29).

El tratamiento de los pacientes con dermatomiositis y cáncer es similar al de los pacientes con cáncer en general; y la asociación no modifica el manejo de la dermatomiositis. Sin embargo, se ha reportado que los pacientes con dermatomiositis y malignidad tienden a tener un peor pronóstico oncológico, puesto que al momento del diagnóstico hay estadios avanzados y no siempre es posible una resolución óptima (17). El manejo quirúrgico satisfactorio usualmente conlleva a resolución de los síntomas de dermatomiositis $(30,31)$. Lo primordial es el diagnóstico temprano de la malignidad en el paciente con dermatomiositis para lograr mejores pronósticos.

La mayoría de casos reportados de dermatomiositis con cáncer colorrectal han sido de sexo femenino (63\%), y el adenocarcinoma es la histología más frecuente en dermatomiositis (96,3\%). En el 77,7 \% de los pacientes se manifestó la dermatomiositis antes que el cáncer colorrectal con niveles elevados de CK. La mejoría inmediata de los síntomas después de la cirugía ocurre en la mitad de los pacientes, los demás mejoran en los primeros meses posquirúrgicos $(31,32,33)$.

En nuestro caso, los síntomas de dermatomiositis se presentaron pocos meses antes del hallazgo del carcinoma en el colon transverso. La resolución de los síntomas musculares y la significativa mejoría en las lesiones cutáneas ocurrió varias semanas después de la resección quirúrgica de la lesión.

\section{CONCLUSIONES}

No se conoce la causa exacta de la relación entre la dermatomiositis y la presencia de malignidad. Se sugiere la presencia de autoantígenos similares en las células neoplásicas, musculoesqueléticas y cutáneas. Hasta una cuarta parte de las dermatomiositis se asocian en su evolución con la presencia de cáncer. $\mathrm{Al}$ momento del diagnóstico de la dermatomiositis, los pacientes con malignidad asociada ya la vienen desarrollando, y esta última generalmente es de evolución lenta. Cuando se encuentran pacientes con dermatomiositis, es de primordial relevancia descartar la presencia de neoplasia maligna subyacente, idealmente en estadios tempranos para lograr intervenciones positivas en cuanto al pronóstico. Usualmente, luego del manejo satisfactorio (resección quirúrgica completa) de la lesión neoplásica, se resuelven los síntomas de dermatomiositis; en caso de recidivas tumorales o aparición de enfermedad metastásica, suelen reaparecer los síntomas de dermatomiositis.

Entre los principales sitios de origen primario potencial se encuentra el tracto digestivo y, en este nivel, las neoplasias más frecuentemente relacionadas son las gástricas y las colorrectales. No debe perderse de vista que el estudio del paciente debe enfocarse según sus factores de riesgo y la clínica asociada.

\section{REFERENCIAS}

1. Zantos D, Zhang Y, Felson D. The overall and temporal association of cancer with polymyositis and dermatomyositis. J Rheumatol. 1994;21(10):1855-9.

2. Barnes BE, Mawr B. Dermatomyositis and malignancy. A review of the literature. Ann Intern Med. 1976;84(1):68-76. https://doi.org/10.7326/0003-4819-84-1-68

3. Callen JP. Relationship of cancer to inflammatory muscle diseases. Dermatomyositis, polymyositis, and inclusion body myositis. Rheum Dis Clin North Am. 1994;20(4):943-53.

4. Hill CL, Zhang Y, Sigurgeirsson B, Pukkala E, Mellemkjaer L, Airio A, Evans SR, Felson DT. Frequency of specific cancer types in dermatomyositis and polymyositis: a population-based study. Lancet. 2001;357(9250):96-100. https://doi.org/10.1016/S0140-6736(00)03540-6

5. Callen JP. Relation between dermatomyositis and polymyositis and cancer. Lancet. 2001 Jan 13;357(9250):85-6. https://doi.org/10.1016/S0140-6736(00)03535-2

6. Maddison P. Cancer types in dermatomyositis and polymyositis. Lancet. 2001 May 5;357(9266): 1443. https://doi.org/10.1016/S0140-6736(00)04587-6

7. Callen JP. Dermatomyositis. Lancet. 2000;355(9197):53-7. https://doi.org/10.1016/S0140-6736(99)05157-0

8. Kamiyama H, Niwa K, Ishiyama S, Takahashi M, Kojima Y, Goto M, Tomiki Y, Higashihara Y, Sakamoto K. Ascending Colon Cancer Associated with Dermatomyositis Which Was Cured after Colon Resection. Case Rep Gastroenterol. 
2016;10(2):338-43.

https://doi.org/10.1159/000447289

9. Zahr ZA, Baer AN. Malignancy in myositis. Curr

Rheumatol Rep. 2011;13(3):208-15.

https://doi.org/10.1007/s11926-011-0169-7

10. Wang J, Guo G, Chen G, Wu B, Lu L, Bao L. Meta-analysis of the association of dermatomyositis and polymyositis with cancer. Br J Dermatol. 2013;169(4):838-47. https://doi.org/10.1111/bjd.12564

11. Casciola-Rosen L, Nagaraju K, Plotz P, Wang K, Levine S, Gabrielson E, Corse A, Rosen A. Enhanced autoantigen expression in regenerating muscle cells in idiopathic inflammatory myopathy. J Exp Med. 2005;201(4):591-601. https://doi.org/10.1084/jem.20041367

12. Levine SM. Cancer and myositis: new insights into an old association. Curr Opin Rheumatol. 2006;18(6):620-4. https://doi.org/10.1097/01.bor.0000245721.02512.77

13. Zampieri S, Valente M, Adami N, Biral D, Ghirardello A, Rampudda ME, Vecchiato M, Sarzo G, Corbianco S, Kern H, Carraro U, Bassetto F, Merigliano S, Doria A. Polymyositis, dermatomyositis and malignancy: a further intriguing link. Autoimmun Rev. 2010;9(6):449-53. https://doi.org/10.1016/j.autrev.2009.12.005

14. Bohan A, Peter JB. Polymyositis and dermatomyositis (first of two parts). N Engl J Med. 1975;292(7):344-7. https://doi.org/10.1056/NEJM197502132920706

15. Dalakas MC, Hohlfeld R. Polymyositis and dermatomyositis. Lancet. 2003;362(9388):971-82. https://doi.org/10.1016/S0140-6736(03)14368-1

16. Fung WK, Chan HL, Lam WM. Amyopathic dermatomyositis in Hong Kong -- association with nasopharyngeal carcinoma. Int J Dermatol. 1998;37(9):659-63. https://doi.org/10.1046/j.1365-4362.1998.00453.x

17. Wakata N, Kurihara T, Saito E, Kinoshita M. Polymyositis and dermatomyositis associated with malignancy: a 30-year retrospective study. Int J Dermatol. 2002;41(11):729-34. https://doi.org/10.1046/j.1365-4362.2002.01648.x

18. Leatham H, Schadt C, Chisolm S, Fretwell D, Chung L, Callen JP, Fiorentino D. Evidence supports blind screening for internal malignancy in dermatomyositis: Data from 2 large US dermatology cohorts. Medicine (Baltimore). 2018;97(2):e9639. https://doi.org/10.1097/MD.0000000000009639

19. Sigurgeirsson B, Lindelöf B, Edhag O, Allander E. Risk of cancer in patients with dermatomyositis or polymyositis. A population-based study. N Engl J Med. 1992;326(6):363-7. https://doi.org/10.1056/NEJM199202063260602

20. Hatada T, Aoki I, Ikeda H, Tamura T, Okada K, Nakai T, Utsunomiya J. Dermatomyositis and malignancy: case report and review of the Japanese literature. Tumori. 1996;82(3):273-5.

21. Stockton D, Doherty VR, Brewster DH. Risk of cancer in patients with dermatomyositis or polymyositis, and followup implications: a Scottish population-based cohort study. Br J Cancer. 2001;85(1):41-5. https://doi.org/10.1054/ bjoc. 2001.1699
22. Chang SH, Park JK, Lee YJ, Yang JA, Lee EY, Song YW, Lee EB. Comparison of cancer incidence among patients with rheumatic disease: a retrospective cohort study. Arthritis Res Ther. 2014;16(4):428. https://doi.org/10.1186/s13075-014-0428-x

23. Yu KH, Kuo CF, Huang LH, Huang WK, See LC. Cancer Risk in Patients With Inflammatory Systemic Autoimmune Rheumatic Diseases: A Nationwide Population-Based Dynamic Cohort Study in Taiwan. Medicine (Baltimore). 2016;95(18):e3540. https://doi.org/10.1097/MD.0000000000003540

24. Ohno S, Oshikawa K, Kitamura S, Saitoh K. Clinicopathological analysis of interstitial pneumonia associated with collagen vascular disease in patients with lung cancer. Nihon Kyobu Shikkan Gakkai Zasshi. 1997;35(12):1324-9.

25. Lakhanpal S, Bunch TW, Ilstrup DM, Melton LJ 3rd. Polymyositis-dermatomyositis and malignant lesions: does an association exist? Mayo Clin Proc. 1986;61(8):645-53. https://doi.org/10.1016/s0025-6196(12)62030-8

26. Basset-Seguin N, Roujeau JC, Gherardi R, Guillaume JC, Revuz J, Touraine R. Prognostic factors and predictive signs of malignancy in adult dermatomyositis. A study of 32 cases. Arch Dermatol. 1990;126(5):633-7.

27. Rose AL, Walton JN. Polymyositis: a survey of 89 cases with particular reference to treatment and prognosis. Brain. 1966;89(4):747-68. https://doi.org/10.1093/brain/89.4.747

28. Dourmishev LA. Dermatomyositis associated with malignancy. 12 case reports. Adv Exp Med Biol. 1999;455:193-9. https://doi.org/10.1007/978-1-4615-4857-7_28

29. Marie I, Hatron PY, Levesque H, Hachulla E, Hellot MF, Michon-Pasturel U, Courtois H, Devulder B. Influence of age on characteristics of polymyositis and dermatomyositis in adults. Medicine (Baltimore). 1999;78(3):139-47. https://doi.org/10.1097/00005792-199905000-00001

30. Nagano $Y$, Inoue $Y$, Shimura T, Fujikawa H, Okugawa Y, Hiro J, Toiyama Y, Tanaka K, Mohri Y, Kusunoki M. Exacerbation of Dermatomyositis with Recurrence of Rectal Cancer: A Case Report. Case Rep Oncol. 2015;8(3):482-6. https://doi.org/10.1159/000439519

31. Gkegkes ID, Minis EE, Iavazzo C. Dermatomyositis and colorectal cancer: a systematic review. Ir J Med Sci. 2018;187(3):615-620. https://doi.org/10.1007/s11845-017-1716-7

32. Dourmishev LA, Draganov PV. Paraneoplastic dermatological manifestation of gastrointestinal malignancies. World J Gastroenterol. 2009;15(35):4372-9. https://doi.org/10.3748/wjg.15.4372

33. Ouahbi H, Benhami M, Nouikh L, Acharfi N, Kelati A, Oualla K, Benbrahim Z, Elmrabet FZ, Arifi S, Mernissi F, Mellas N. Dermatomyosite et cancer rectal: à propos d'un cas avec revue de la littérature. Pan Afr Med J. 2019;33:122. https://doi.org/10.11604/pamj.2019.33.122.14509 